\title{
芳基偶氮砜的芳基化及砜基化研究进展
}

\author{
孟 娜 $\dagger, a$ \\ 刘启顺 ${ }^{\dagger}, a$ \\ 刘瑞生 ${ }^{a}$ \\ 吕玉芬*,a \\ 赵晓辉*,b魏 伟*,,$b$ \\ ( ${ }^{a}$ 曲阜师范大学化学与化工学院 山东曲阜 273165) \\ ( $b$ 中国科学院西北高原生物研究所 中国科学院藏药研究重点实验室 青海西宁 810008)
}

\begin{abstract}
摘要 芳基和砜基化合物在合成化学、药物化学、材料化学等领域具有广泛的应用价值. 因此, 其清洁、高效的构建 方法备受人们的关注. 芳基偶氮砜在光、电或热条件下可以发生 $\mathrm{C}-\mathrm{N}$ 和 $\mathrm{S}-\mathrm{N}$ 键均裂, 产生芳基和砜基自由基，进而 发生芳基化或砜基化反应选择性构建芳基或砜基化合物. 归纳总结了近年来芳基偶氮砜参与的芳基化及砜基化反应最 新研究进展，重点介绍了其合成方法及反应机理，并对该领域的未来发展进行了展望.
\end{abstract}

关键词 芳基偶氮砜; 光催化; 电催化; 芳基化; 砜基化

\section{Recent Advances in Arylations and Sulfonylations of Arylazo Sulfones}

\author{
Meng, $\mathrm{Na}^{\dagger, a}$ \\ Liu, Qishun ${ }^{\dagger, a}$ \\ Liu, Ruisheng ${ }^{a}$ \\ Lü, Yufen*,a \\ Zhao, Xiaohui*,b \\ Wei, Wei*,a,b
}

( ${ }^{a}$ School of Chemistry and Chemical Engineering, Qufu Normal University, Qufu, Shandong 273165)

( ${ }^{b}$ Key Laboratory of Tibetan Medicine Research, Chinese Academy of Sciences, Northwest Institute of Plateau Biology, Chinese Academy of Sciences, Xining, Qinghai 810008)

\begin{abstract}
Aromatic ring- and sulfone-containing compounds are widely used in the fields of synthetic chemistry, medicinal chemistry and material chemistry. Therefore, it is of great significance to develop a simple, green and efficient method to construct aryl and sulfonyl compounds. Arylazo sulfones can be used as arylation or sulfonylation reagents to access aryl and sulfonyl compounds via hemolytic cleavage of $\mathrm{C}-\mathrm{N}$ and $\mathrm{N}-\mathrm{S}$ bonds to generate aryl radical and sulfonyl radical. In this review, the recent research progress of arylations and sulfonylations using arylazo sulfones is summarized. The synthetic methods and the related reaction mechanisms are highlighted, and an outlook of this research field is also given.

Keywords arylazo sulfones; photocatalysis; electrocatalytic; arylations; sulfonylations
\end{abstract}

芳香环是一种非常重要的有机结构单元, 很多天然 产物 ${ }^{[1}$ 和药物分子 ${ }^{[2]}$ 中往往都包含此结构骨架, 它们在 杀菌、抗病毒 ${ }^{[3]}$ 以及材料合成 ${ }^{[4]}$ 方面也有着广泛的应用. 另一方面, 砜基官能团也是一种非常重要的化学基团, 其广泛分布于一些生物活性分子和天然产物 ${ }^{[5]}$ 中, 在抗 菌 ${ }^{[6]}$ 、杀虫 ${ }^{[7]}$ 和抑制人体免疫缺陷病毒 1 型(HIV-1)逆转 录酶 ${ }^{[8}$ 等方面发挥着重要作用. 因此, 发展绿色、简便、 高效的芳基化和砜基化方法具有重要意义. 芳基偶氮砜 是一种相对稳定且具有颜色的固体化合物, 可以通过芳 胺与氢氟酸和亚硝酸钠反应制得芳基重氮盐, 然后其再
与亚磺酸钠反应简便合成 ${ }^{[9]}$. 在光照、加热或者电催化 条件下, 芳基偶氮砜可以发生 $\mathrm{C}-\mathrm{N}$ 和 $\mathrm{S}-\mathrm{N}$ 键均裂分 解产生芳基和砜基自由基或离子, 在有机合成中用作芳 基化和砜基化反应试剂 ${ }^{[10]}$. 由于芳基偶氮砜参与的芳 基化和砜基化反应条件温和，因此其在有机合成中的应 用吸引了化学家的广泛关注. 近年来, 国内外多个课题 组开发了一系列光照、加热或电催化下芳基偶氮砜参与 的芳基化及砜基化反应(Scheme 1). 本文综述了芳基偶 氮砜参与有机反应的最新研究进展, 对不同的芳基化及 砜基化反应类型和机理进行了分类探讨和归纳总结，期

\footnotetext{
*Corresponding authors. E-mail: weiweiqfnu@163.com; xhzhao@nwipb.cas.cn; yufenlv06@163.com Received July 7, 2021; revised July 29, 2021; published online August 9, 2021.

Project supported by the Youth Innovation and Technology Project of Higher School in Shandong Province (No. 2019KJC021), and 2018 CAS "Light of West China" Program.

山东省高校青创科技计划(No. 2019KJC021)、2018 中国科学院“西部之光”人才培养引进计划资助项目.

$\dagger$ 共同第一作者(These authors contributed equally to this work).
} 


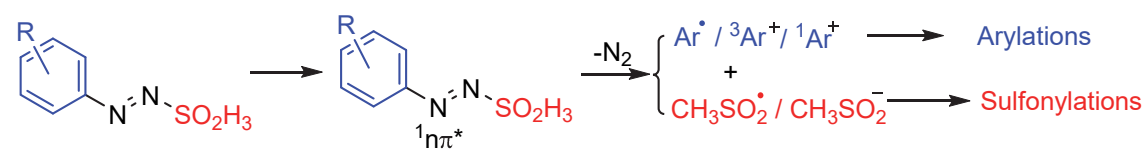

图式 1 芳基偶氮砜的芳基化和砜基化反应应用

Scheme 1 Application of arylazo sulfones for arylations and sulfonations

望对未来发展芳基偶氮砜的芳基化及砜基化反应起到 一定的促进作用.

\section{1 芳基偶氮砜参与的芳基化反应}

\section{1 可见光介导下芳基偶氮砜参与的芳基化反应}

在过去一段时间里, 芳基化反应研究已经取得了重 大进步，化学家们利用芳基重氮盐 ${ }^{[11]}$ 、芳基卤代物 ${ }^{[12] 、}$ 芳基磺酰氯 ${ }^{[13]}$ 、二芳基碘盐 ${ }^{[14]}$ 、芳基嗍酸 ${ }^{[15]}$ 和芳香环作 芳基化试剂, 在过渡金属催化、光催化剂催化氧化条件 下可以将芳基引入有机分子, 构建一系列芳基化合物 (Scheme 2). 虽然这些方法可以在一些底物上引入芳基, 但这些方法往往需要使用 $\mathrm{Pd} 、 \mathrm{Ru}$ 等不可再生的贵金属、 光催化剂或氧化剂, 容易造成环境污染和资源浪费. 因 此, 开发一种清洁、简便的合成方法显得尤为重要.

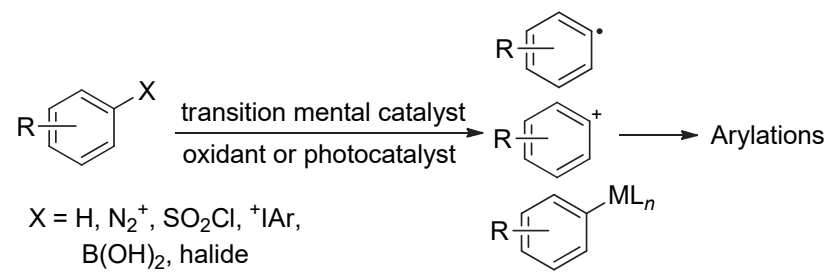

图式 2 常见的芳基化合物合成方法

Scheme 2 General methods for the synthesis of aryl compounds

近年来, 可见光能作为清洁、可再生的能源在有机 合成反应中得到了广泛应用 ${ }^{[16]} .2016$ 年, Fagnoni 课题 组 ${ }^{[17]}$ 利用芳基偶氮砜自身可以吸收可见光能裂解生成 芳基自由基的特性, 发展了在可见光照射下芳基偶氮砜 1 与芳香环 2 和芳香杂环 $\mathbf{4}$ 的芳基化反应. 该反应利用 乙腈和水 $(V: V=9: 1)$ 作为混合溶剂, 直接在阳光照射 下就可以简便合成相应的联芳基化合物 3 和 5 (Scheme $3)$.

该芳基化反应不需要任何金属等添加剂，操作方 便, 反应条件温和, 时间短, 产率好. 各种芳香杂环如 呋喃、吡咯、噻吩以及富电子芳环都可以很好地兼容该 反应体系, 为各种联芳基化合物提供了一种绿色、高效 的合成方法. 可能的反应机理如 Scheme 4 所示, 芳基偶 氮砜在可见光照射下到达激发态 $\mathbf{6}$ 和 7, 由于吸收能量 的不同，可以有两种不同的跃迁方式. 当发生 $n-\pi$ 跃迁 时, 产生芳基自由基 9 与砜基自由基 10, 芳基自由基 9 加成到杂芳香环 $2 \mathrm{a}$ 上, 产生中间体 11, 其在砜自由基

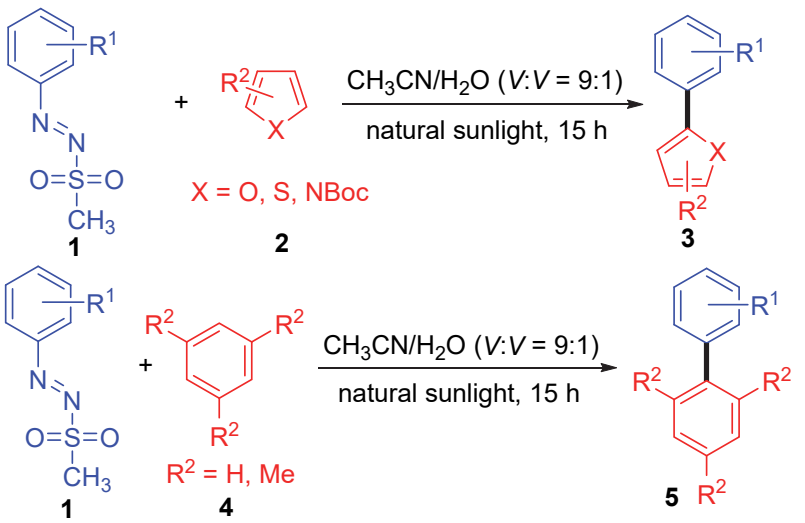

图式 3 光促进芳基偶氮砜与芳香环/芳香杂环的芳基化反应 Scheme 3 Visible-light-induced arylation reaction of arylazo sulfones with aromatic/heteroaromatic ring

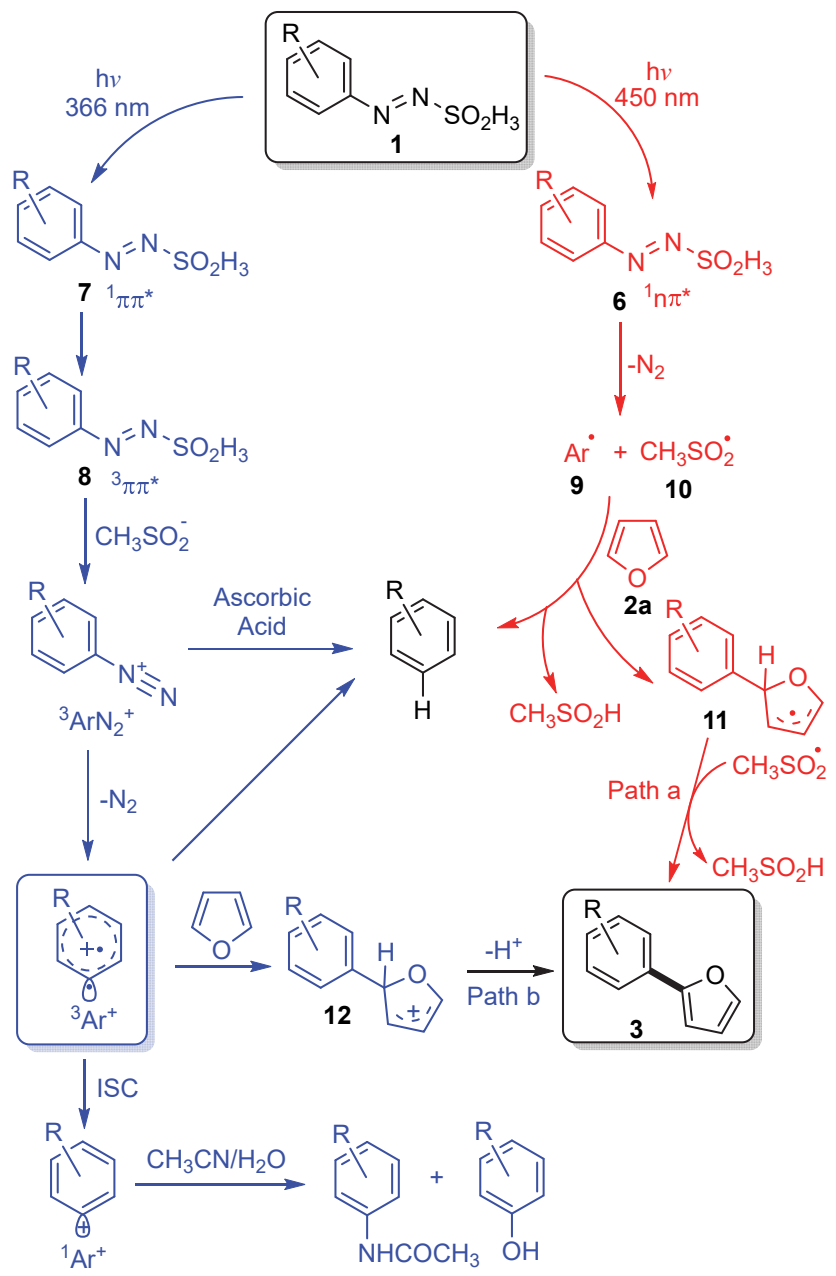

图式 4 芳基偶氮砜与芳香环/芳香杂环的芳基化反应机理

Scheme 4 Reaction mechanism for arylation reaction of arylazo sulfones with aromatic/heteroaromatic ring 
10 作用下生成了相应的联芳基化合物 3 . 当发生 $\pi-\pi$ 跃 迁时, 产生芳基正离子与砜基负离子, 芳基正离子加成 到杂芳香环底物 $2 \mathrm{a}$ 上生成碳正离子中间体 12 , 其进一 步发生消除反应生成了目标产物 3.

随后, 在可见光照射下, 以芳基偶氮砜作为芳基化 试剂得到广泛应用和推广. 2017 年, Fagnoni 课题组 ${ }^{[18]}$ 又 利用芳基偶氮砜 1 与烯丙基砜 13 为原料, 在可见光照射 下，通过脱砜化在烯丙基位置引入了芳基. 该反应中选 用乙腈和水 $(V: V=9: 1)$ 为混合溶剂, 在没有添加任何 过渡金属试剂和光催化剂的条件下, 采用波长 $\lambda$ 为 410 $\mathrm{nm}$ 的 $1 \mathrm{~W}$ 发光二极管(LED)灯照射合成了一系列烯丙 基取代的芳烃. 该反应对不同取代的芳基偶氮砜进行了 拓展, 研究发现芳环上带有吸电子基时反应效果较好, 芳环上带有给电子基时反应效果较差(Scheme 5).

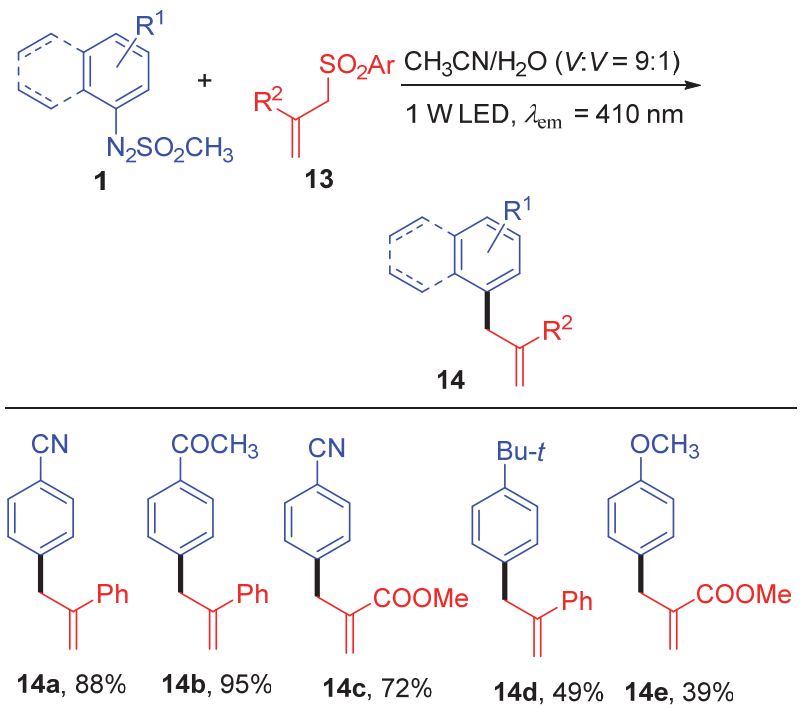

图式 5 芳基偶氮砜和烯丙基砜反应构建烯丙基取代芳烃 Scheme 5 Reaction of arylazo sulfones with allyl sulfones to construct allyl arenes

2017 年, Bandini 团队 ${ }^{[19]}$ 利用芳基偶氮砜与芳基硼 酸反应, 在 $7 \mathrm{~W}$ 蓝光照射下实现了芳环 $\mathrm{C}-\mathrm{C}$ 键的偶联 反应构建一系列联苯类化合物(Scheme 6). 该反应采用

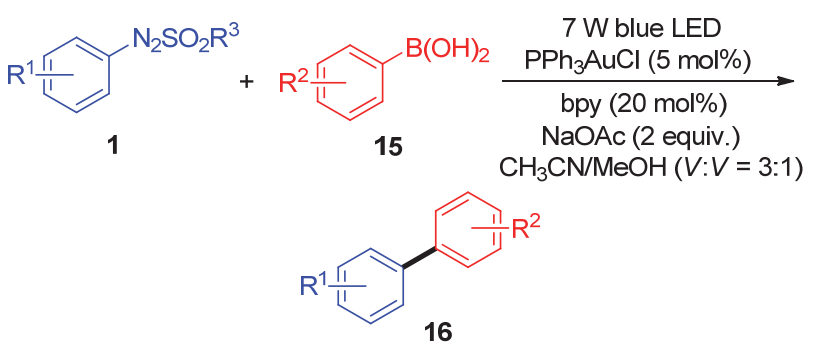

图式 6 光诱导芳基偶氮砜和芳基硼酸反应构建联苯类化合 物

Scheme 6 Visible-light-induced reaction of arylazo sulfones with arylboronic acids to construct (hetero)biaryls
$\mathrm{PPh}_{3} \mathrm{AuCl}$ (5 mol\%)作为催化剂, 2,2'-联吡啶(20 mol\%)为 配体, 2 equiv.的醋酸钠为碱, 乙腈与甲醇 $(V: V=3: 1)$ 为混合溶剂, 在常温条件下就可有效构建各种联苯类化 合物．该合成方法具有操作步骤简单、反应产率好等优 点.

该反应机理如 Scheme 7 所示，该反应中芳基偶氮 砜在可见光照射下分解为芳基自由基 9 和砜基自由基 10, 同时释放出氮气. 芳基自由基 9 氧化加成到 $[\mathrm{Au}(\mathrm{I})]$ 上生成[ $\mathrm{Au}(\mathrm{II})]$ 中间体 17, 中间体 17 被砜基进一步氧化 得到 $[\mathrm{Au}(\mathrm{II})]$ 中间体 18. 此时芳基硼酸在碱的作用下使 得 $\mathrm{C}-\mathrm{B}$ 键极性增强, 从而促进了其与 18 的相互作用, 生成了中间体 19. 最后, 中间体 19 通过还原消除反应 释放出联苯产物 16 并完成催化剂循环.

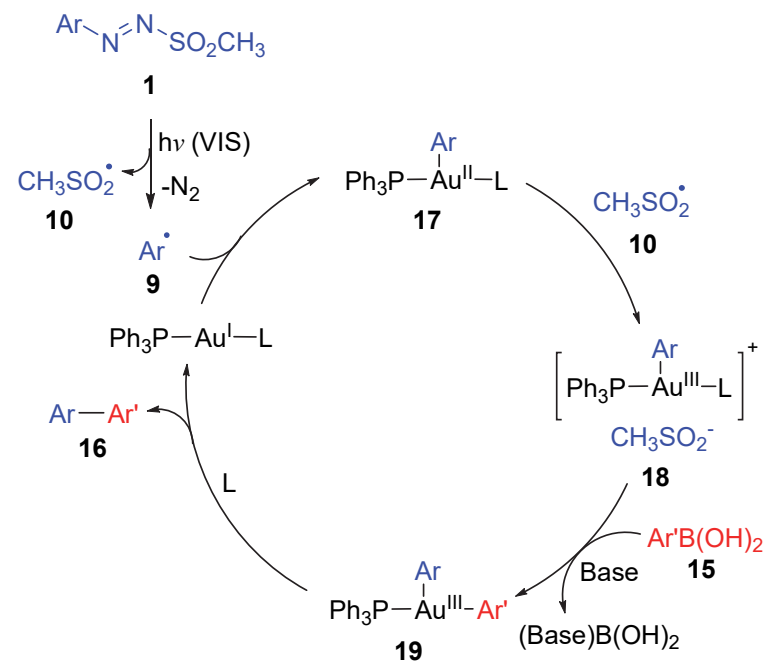

图式 7 芳基偶氮砜和芳基硼酸反应机理

Scheme 7 Reaction mechanism for arylation reaction of arylazo sulfones with arylboronic acids

2017 年, Fagnoni 团队 [20]采用芳基偶氮砜与异腈的 加成水解反应合成了一系列芳香酰胺类化合物. 该合成 方法利用波长 $\lambda$ 为 $450 \mathrm{~nm}$ 的 $1 \mathrm{~W}$ LED 灯作为反应光源, 2 equiv. 碳酸氢钠为碱, 乙腈和水 $(V: V=9: 1)$ 为混合溶 剂. 随后 Fagnoni 团队 ${ }^{[20]}$ 在最优条件下，对不同的芳基 偶氮砜与异腈进行了拓展，将不同取代基的芳基偶氮砜 在最优条件下与叔丁基异腈反应，得到了众多芳香酰胺 化合物. 如 Scheme 8 所示, 偶氮砜芳环上带有吸电子基 才时反应效果较好，芳环上带有给电子基团时，反应效 果较差. 值得注意的是, 杂芳环如吡啶偶氮砜也能参与 反应，得到 $25 \%$ 收率的杂芳酰胺 $21 \mathrm{k}$. 此外，各种脂肪 取代的异腈都可很好的适用于该反应. 该反应在没有过 渡金属催化剂、光催化剂或氧化剂的条件下就可以有效 进行，条件温和，操作简单，底物适用性广，产物产率 高，为芳香酰胺提供了一种温和、有效的合成策略. 

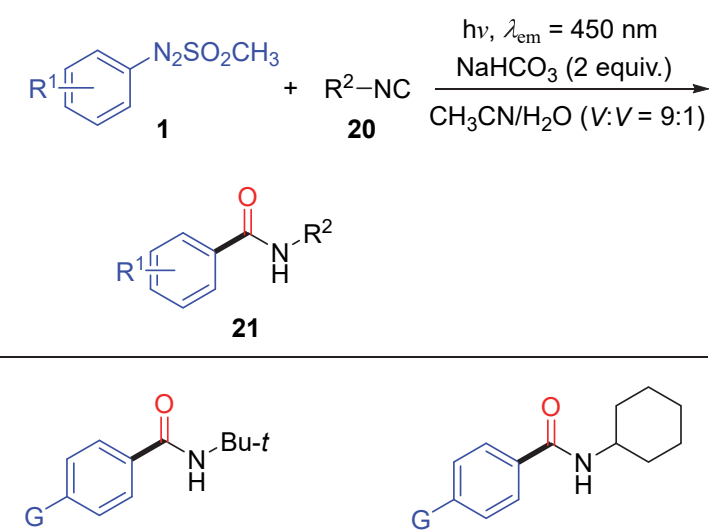

21a, $\mathrm{G}=\mathrm{COCH}_{3}, 68 \%$

21b, $\mathrm{G}=\mathrm{NO}_{2}, 98 \%$

21c, $\mathrm{G}=\mathrm{CN}, 70 \%$

21d, $\mathrm{G}=\mathrm{OMe}, 77 \%$

21e, $\mathrm{G}=t-\mathrm{Bu}, 47 \%$<smiles>CN(C)c1ccc(C(=O)NC(C)(C)C)cc1</smiles>

21j, $38 \%$<smiles>O=C(NC1CCCCC1)c1ccc(Cl)cc1</smiles>

21f, $\mathrm{G}=\mathrm{COCH}_{3}, 71 \%$ 21g, $\mathrm{G}=\mathrm{NO}_{2}, 96 \%$

21h, $\mathrm{G}=\mathrm{CN}, 81 \%$

21i, G = OMe, $29 \%$<smiles>CC(C)(C)NC(=O)c1cccnc1</smiles>

21k, 25\%<smiles>[Z10]S(=O)(=O)O[Na]</smiles>

图式 8 可见光诱导下芳基偶氮砜和异腈反应构建取代的芳 香酰胺

Scheme 8 Visible-light-induced reaction of arylazo sulfones with isonitriles to construct aromatic amides

自由基捕捉实验证实该反应经历自由基的反应进 程. 反应机理如 Scheme 9 所示, 首先芳基偶氮砜 1 在可 见光照射下发生 $n-\pi$ 跃迁到达电子激发态 6.6 通过裂解 产生芳基自由基 9 和砜基自由基 10, 同时释放出氮气. 随后, 芳基自由基 9 与异腈 $\mathbf{2 0}$ 进行自由基加成反应生成 亚胺碳自由基 22. 亚胺碳自由基 22 与砜基自由基经过 单电子转移得到氮正离子. 最后, 氮正离子经水解反应 生成相应的芳香酰胺 21.

2018 年, Fagnoni 和团队成员 ${ }^{[21]}$ 在阳光照射下利用 芳基偶氮砜和 1,1-二芳基烯烃的偶联反应实现了三芳基 烯烃化合物的合成(Scheme 10). 与传统合成方法相比, 该反应不需要添加任何光催化剂或者金属试剂, 在太阳 光下就可以有效进行. 作者在最优条件下通过对不同取 代基底物的普适性考察发现芳环上无论带有吸电子基 团或者给电子基团，反应都能够很好的进行. 一些杂环 芳基偶氮砜如吡啶环的偶氮砜也能在可见光下实现与 烯烃 $\mathrm{C}-\mathrm{C}$ 键的偶联反应. 该方法为三芳基烯烃化合物 提供了一种清洁、高效的合成策略.
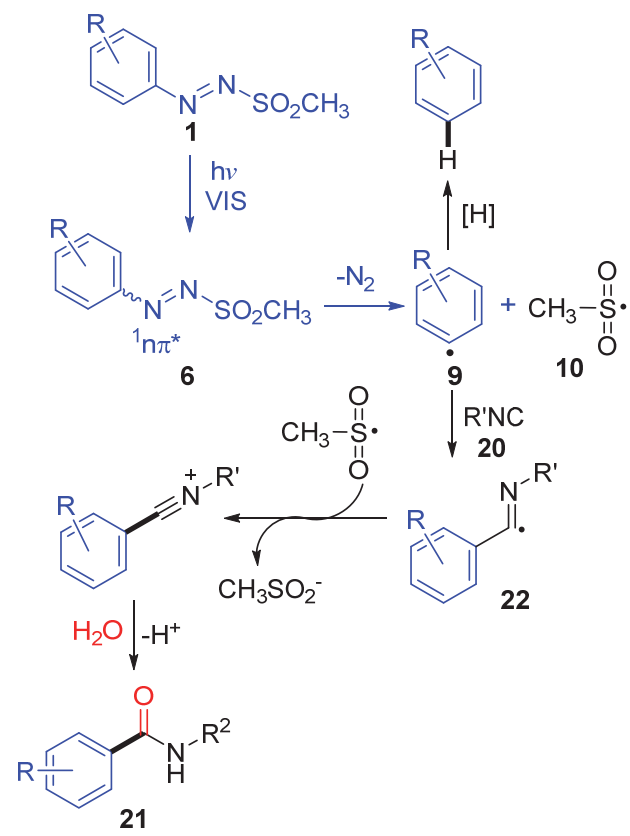

图式 9 芳基偶氮砜和异腈反应构建芳香酰胺的反应机理

Scheme 9 Reaction mechanism for the synthesis of aromatic amides from arylazo sulfones and isonitriles

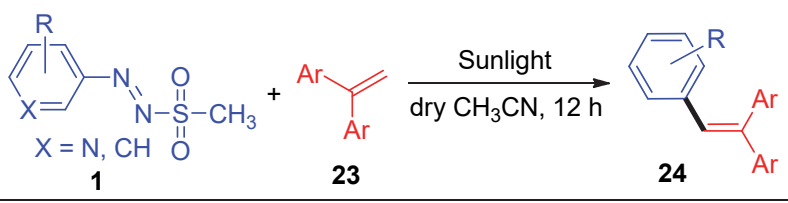<smiles>[R]1ccccc1C=C(c1ccccc1)c1ccccc1</smiles>

24a, $R=4-\mathrm{CN}, 80 \%$

24b, $\mathrm{R}=4-\mathrm{COCH}_{3}, 83 \%$

24c, $\mathrm{R}=4-\mathrm{NO}_{2}, 56 \%$

24d, $R=4-\mathrm{COOMe}, 56 \%$

24e, $R=4-M e, 89 \%$

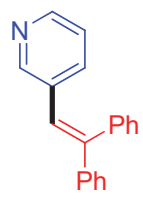

24f, $49 \%$

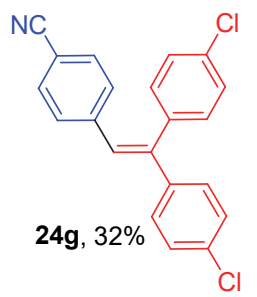

图式 10 光介导下芳基偶氮砜和 1,1-二芳基乙烯反应构建三 芳基乙烯

Scheme 10 Visible-light-induced reaction of arylazo sulfones with 1,1-diarylethylenes to construct triarylethylenes

2018 年, 方浩课题组 ${ }^{[22]}$ 利用芳基偶氮砜在可见光 照射下可以分解生成芳基自由基的特性，发展了一种室 温下可见光诱导芳基偶氮砜嗍化反应，合成了一系列芳 基硼酸酯的方法(Scheme 11). 该课题组选用乙腈和水 $(V: V=9: 1)$ 为混合溶剂, $30 \mathrm{~W}$ 蓝光 LED 灯为光源, 在 氮气氛围下实现了联嗍酸频那醇酯与芳基偶氮砜的偶 联反应. 该方法不需要任何光催化剂或添加剂, 不仅为 芳基硼酸酯提供了一种绿色、高效的合成方法，也为 $\mathrm{C}$ - B 键的构建提供了一种新的反应策略. 

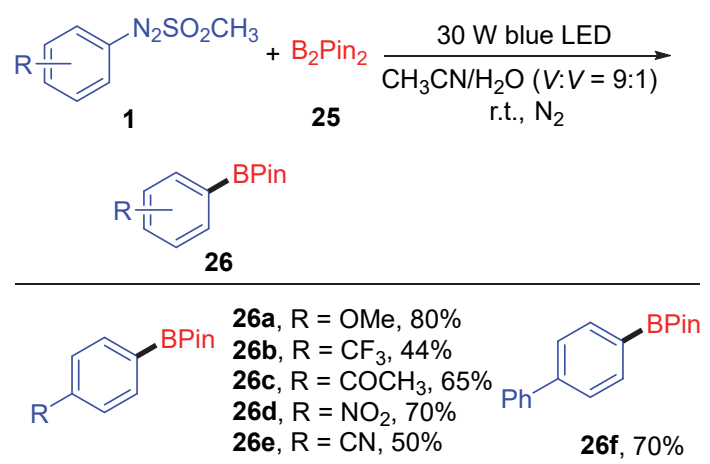<smiles>FC(F)(F)c1cccc(Br)c1</smiles>

26g, $44 \%$<smiles>O=C(O)c1ccc(Br)cc1</smiles>

$26 \mathbf{i}, 60 \%$

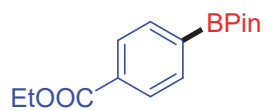

26h, $75 \%$

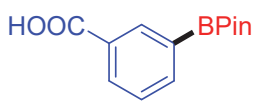

26j, $40 \%$
图式 11 光介导下芳基偶氮砜和联嗍酸频那醇酯反应

Scheme 11 Visible-light-induced reaction of arylazo sulfones and $\mathrm{B}_{2}$ pin 2

其反应机理如 Scheme 12 所示, 首先芳基偶氮砜 $\mathbf{1}$ 在可见光照射下吸收能量发生 $n-\pi$ 跃迁到达电子激发态 6. 然后, 芳基偶氮砜均裂产生芳基自由基 9 和砜基自由 基，同时释放出氮气. 最后，芳基自由基 9 与联硼酸频 那醇酯 25 反应形成芳基喼酸酯化合物 26.

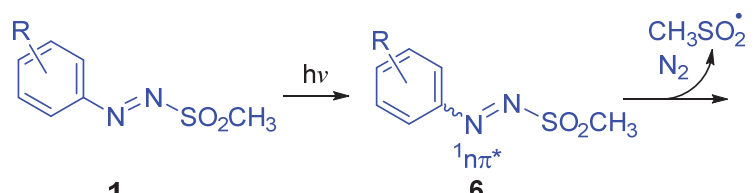

1

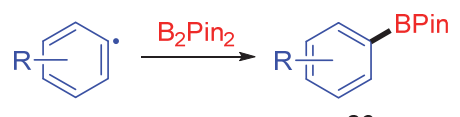

9

26

图式 12 光介导下芳基偶氮砜和联嗍酸频那醇酯反应构建芳 基硼酸酯的反应机理

Scheme 12 Reaction mechanism for the visible light induced synthesis of arylboronates form arylazo sulfones and bis(pinacolato)diboron

喹喔啉酮作为一种重要的杂环化合物骨架广泛存 在于生物活性分子和药物分子中, 在药物化学方面得到 十分广泛的应用 ${ }^{[23]}$. 近年来, 特别是 3 位取代喹喔啉酮 吸引人们的广泛合成兴趣 ${ }^{[24]} .2018$ 年, Kim 教授课题 组 ${ }^{[25]}$ 发展了可见光诱导下芳基偶氮砜与喹喔啉酮 C(3) 位芳基化反应，有效构建了一系列 3-芳基取代喹喔啉酮 化合物. 该反应采用 $\lambda_{\max }=455 \mathrm{~nm}$ 的 $5 \mathrm{~W} \mathrm{LED}$ 灯为光 源, 不需要任何添加剂, 在乙腈和水体积比 $9: 1$ 混合溶
剂中就可以高效得到相应的 3-芳基取代喹喔啉酮 (Scheme 13). 研究发现该反应底物适应范围宽泛, 各种 供电子基或吸电子基取代的芳基偶氮砜都可以很好地 适用该反应条件，以中等至较好的收率得到 3-芳基取代 喹喔啉酮. 此外, 氮氢喹喔啉酮和各种氮取代喹喔啉酮 也都能很好兼容该反应.

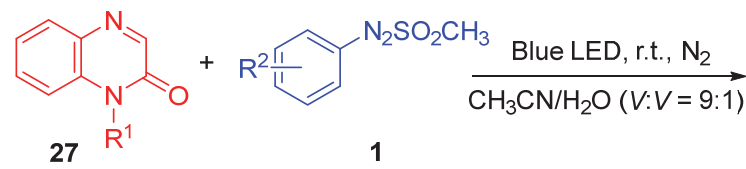

$27 R^{1}$<smiles>[R]c1ccc(-c2nc3ccccc3[nH]c2=O)cc1</smiles>

$\mathrm{R}^{1} \mathbf{2 8}$

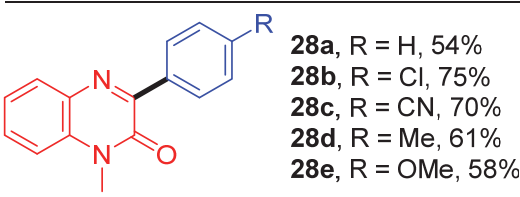<smiles>O=c1[nH]c2ccccc2nc1-c1ccccc1</smiles>

28f, $56 \%$<smiles>CCn1c(=O)c(-c2ccccc2)nc2ccccc21</smiles>

28g, $59 \%$<smiles>O=c1c(-c2ccccc2)nc2ccccc2n1Cc1ccccc1</smiles>

28h, $79 \%$<smiles>CCOC(=O)Cn1c(=O)c(-c2ccccc2)nc2ccccc21</smiles>

28i, $61 \%$
图式 13 光诱导芳基偶氮砜与喹喔啉酮芳基化反应 Scheme 13 Visible-light-induced arylations of quinoxalin2(1H)-ones with arylazo sulfones

可能的反应机理如 Scheme 14 所示，首先芳基偶氮 砜 1 在可见光照射下吸收能量发生 $n-\pi$ 跃迁到达电子激 发态 6. 其通过裂解产生芳基自由基 9 . 随后，芳基自由 基 9 与喹喔啉酮 27 进行 $\mathrm{C}(3)$ 位自由基加成反应得到了 氮自由基中间体 29. 最后, 氮自由基中间体 29 在砜基 作用下脱去氢离子, 就得到了相应的 3-芳基取代喹喔啉 酮产物 28.<smiles>[R]n1c(=O)c([Al])nc2ccccc21</smiles>

图式 14 芳基偶氮砜与喹喔啉酮芳基化反应可能的机理 Scheme 14 The possible reaction mechanism for the arylations of quinoxalin-2 $(1 H)$-ones with arylazo sulfones

2019 年, 邱頔、赵霞和 Protti 课题组 ${ }^{[26]}$ 利用可见光 诱导芳基偶氮砜与六甲基二锡烷进行芳基化反应合成 
了芳基锡化合物. Protti 团队选用在 $24 \mathrm{~W}$ 蓝光 LED 灯为 可见光光源，乙腈为反应溶剂，在氮气保护下芳基偶氮 砜与六甲基二锡烷反应合成了多种芳基锡化合物 (Scheme 15). 该团队对不同取代基的芳基偶氮砜进行 了底物拓展, 研究发现各种取代基的芳环, 包括一些杂 环芳烃如喹啉、吡啶都能生成相应的芳基锡化合物.

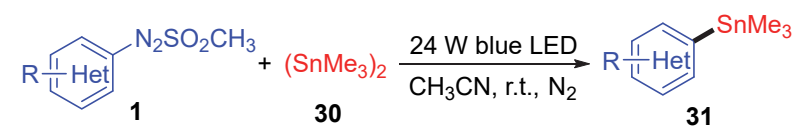

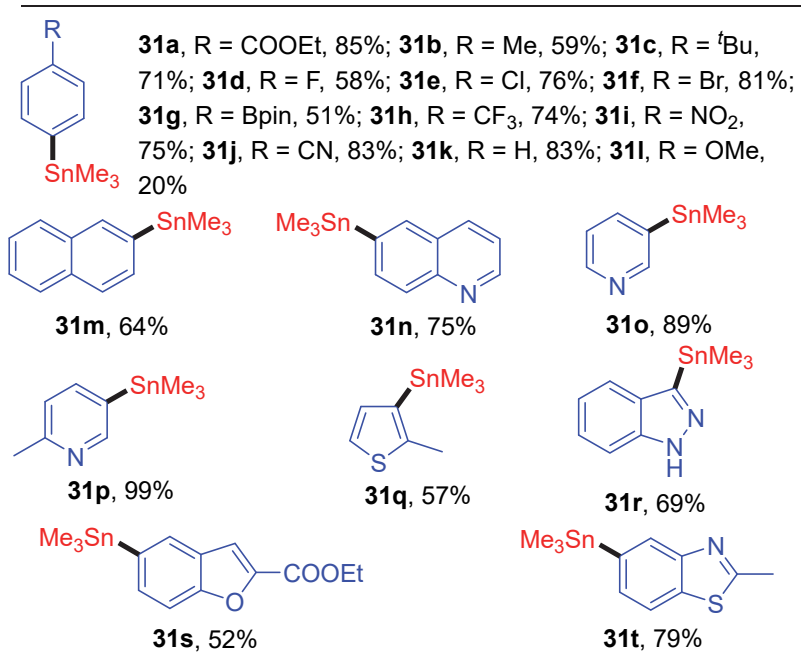

图式 15 光诱导下芳基偶氮砜和六甲基二锡烷反应构建芳基 锡化合物

Scheme 15 Visible-light-induced reaction of arylazo sulfones with hexaalkyldistannanes to construct (hetero)arylstannanes

控制实验表明该反应经历自由基反应历程. 相应的 反应机理如 Scheme 16 所示, 在可见光照射下, 芳基偶 氮砜分解生成了芳基自由基 9 和砜基自由基 $\mathbf{1 0}$. 一种反 应路径为芳基自由基 9 与六甲基二锡烷反应生成芳基锡 化合物 31. 同时, 生成一分子锡基自由基 32. 另一个可 能的反应路径是, 锡基自由基 32 直接与芳基自由基反

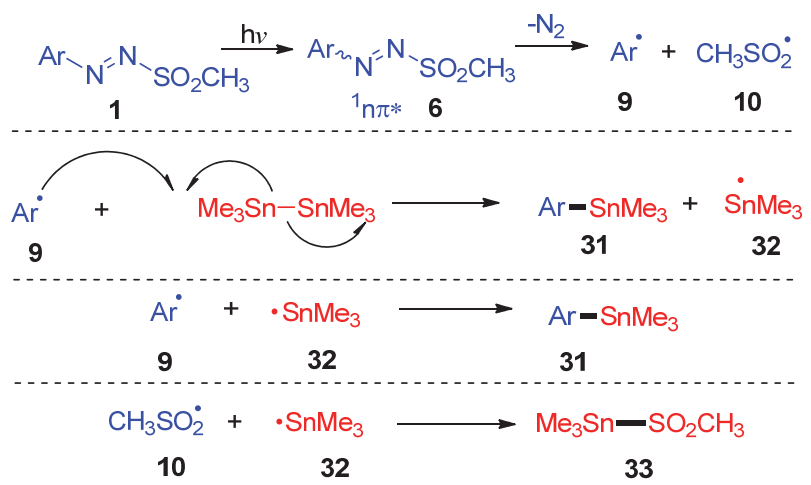

图式 16 光诱导下芳基偶氮砜与六甲基二锡烷反应构建芳基 锡化合物反应机理

Scheme 16 Reaction mechanism for the arylations of hexaalkyldistannanes with arylazo sulfones to construct (hetero)arylstannanes
应得到芳基锡化合物. 锡基自由基与砜自由基反应生成 砜基锡化合物 33 .

2019 年, 邱頔课题组 ${ }^{[27]}$ 开发了可见光诱导下芳基 偶氮砜与亚磷酸酯的芳膦化反应. 该方法利用 $24 \mathrm{~W}$ 蓝 光 LED 灯为反应光源, 乙腈作为反应溶剂, 在氮气条件 下完成了多种芳基取代磷酸酯的构建. 该合成方法避免 使用金属试剂以及光催化剂, 为 $\mathrm{C}-\mathrm{P}$ 键的构建提供了 一种新的合成策略. 通过对不同底物的拓展研究发现反 应具有良好的底物适用性，各种不同取代基的芳(杂)环 都能很好兼容该反应，以中等至较好的收率得到相应的 芳基磷酸酯(Scheme 17).

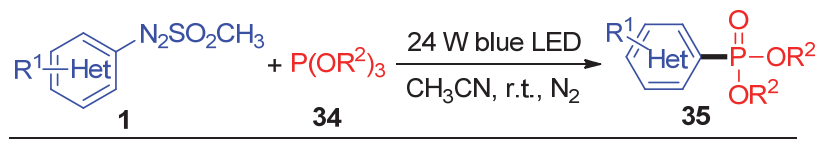

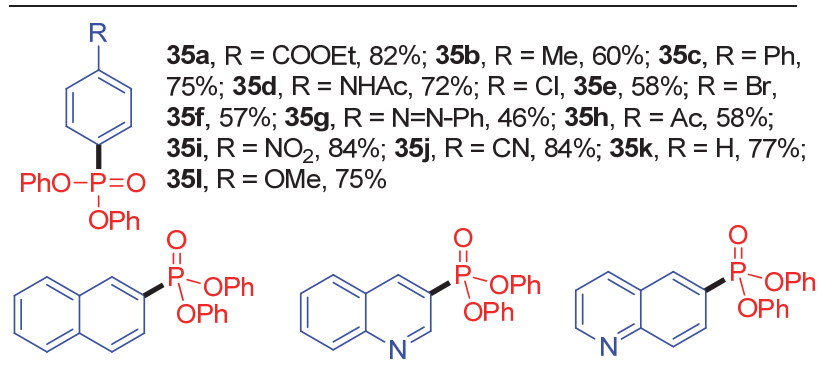

$35 \mathrm{~m}, 64 \%$

$35 n, 54 \%$

$350,43 \%$<smiles>O=P(O)(c1ccccc1)c1cccnc1</smiles>

$35 p, 80 \%$

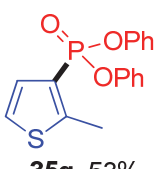
$35 q, 53 \%$<smiles>O=P(c1ccccc1)(c1ccccc1)c1n[nH]c2ccccc12</smiles>

$35 r, 37 \%$<smiles>CCOC(=O)c1cc2cc(P(=O)(c3ccccc3)c3ccccc3)ccc2o1</smiles><smiles>Cc1nc2cc(P(=O)(O[Ga])c3ccccc3)cnc2s1</smiles>

图式 17 光诱导芳基偶氮砜和亚磷酸酯反应构建芳基磷酸酯 Scheme 17 Visible-light-induced reaction of arylazo sulfones with phosphites to construct arylphosphonates

可能的反应机理如 Scheme 18 所示, 在可见光照射 下，芳基偶氮砜分解生成了芳基自由基 9 和砜基自由基 10, 同时释放出氮气. 生成的芳基自由基 9 与亚磷酸酯 分子反应生成了中间体 36. 随后，中间体 36 被甲基砜 自由基氧化生成了目标产物芳基磷酸酯化合物 35. 同 时，反应中检测到芳基自由基氢化的产物，进一步证实 芳基自由基的存在.

亚砜是极有价值的含硫有机化合物, 它不仅大量存 在于天然产物和药理活性化合物中, 而且还常常用于构 建各种重要药物分子. 其传统合成方法主要依赖于硫醚 的氧化，涉及使用金属试剂和化学计量的高价碘或过氧 化物氧化剂. 2019 年, 魏伟课题组 ${ }^{[28]}$ 利用可见光诱导策 


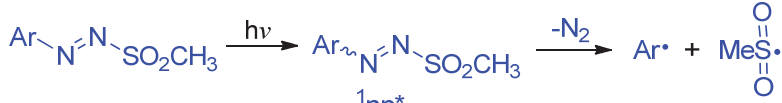

$$
\begin{aligned}
& 1 \\
& { }^{1} \mathrm{np}^{*} 6 \\
& 9 \quad 10
\end{aligned}
$$

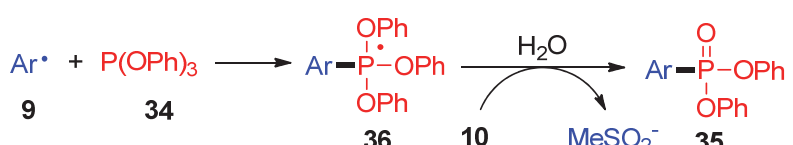

$$
\begin{aligned}
& \mathrm{Ar} \cdot \stackrel{[\mathrm{H}]}{\longrightarrow} \mathrm{Ar}-\mathrm{H} \\
& 9
\end{aligned}
$$

图式 18 芳基偶氮砜与亚磷酸酯反应构建芳基磷酸酯的反应

机理

Scheme 18 Reaction mechanism for arylations of phosphites with arylazo sulfones to construct aryl phosphonates

略, 实现了无光催化剂和外加氧化剂条件下芳基偶氮砜 与硫醇氧化偶联反应构建不对称亚砜的方法. 该反应利 用 $3 \mathrm{~W}$ Blue LED 灯作为反应的光源, 乙腈和水体积比 $1: 1$ 作为反应溶剂, 空气为氧化剂, 一锅法实现了 $\mathrm{C}-$ $\mathrm{S}$ 和 $\mathrm{S}=\mathrm{O}$ 键的构建. 该反应底物范围广且官能团容忍 性好, 为亚砜提供了一种简便、绿色的合成方法.
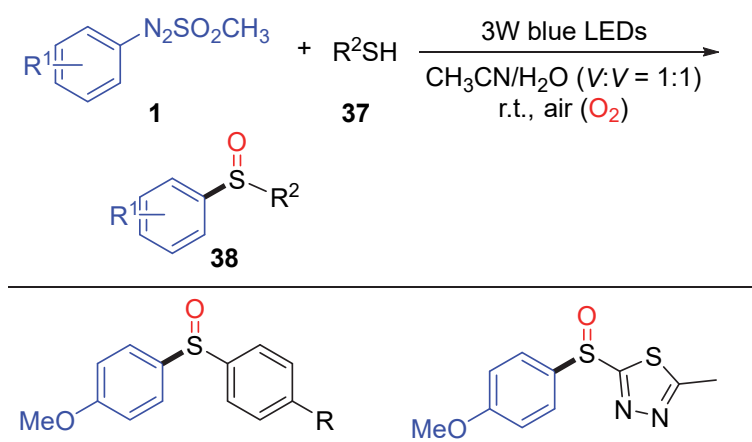

38a, $\mathrm{R}=\mathrm{H}, 79 \%$; 38b, $\mathrm{R}=\mathrm{Me}, 82 \%$;

38c, $R=O M e, 81 \% ; 38 d, R=F, 77 \%$;

38e, $\mathrm{R}=\mathrm{Cl}, 68 \% ; \mathbf{3 8 f}, \mathrm{R}=\mathrm{OH}, 81 \%$<smiles>COc1ccc(S(=O)c2nc3ccccc3s2)cc1</smiles>

$38 \mathrm{~h}, 47 \%$<smiles>CCCCS(=O)c1ccc(OC)cc1</smiles>

38 j, $80 \%$<smiles>COc1ccc(S(=O)Cc2ccccc2)cc1</smiles>

38i, $72 \%$<smiles>COC(=O)CS(=O)c1ccc(OC)cc1</smiles>

图式 19 光诱导芳基偶氮砜和硫酚反应构建不对称亚砜化合 物

Scheme 19 Visible-light-induced reaction of arylazo sulfones and thiols to construct unsymmetrical sulfoxides

基于一系列控制实验, 作者提出一个可能的反应机 理(Scheme 20), 首先, 芳基偶氮砜在可见光照射下产生 芳基自由基 9. 硫酚在空气氧化下产生巯基自由基 39, 其自聚得到二硫醚 40. 然后, 产生的芳基自由基 9 与二 硫醚 40 进行偶联反应得到硫醚 41. 最后, 硫醚 41 被单
线态氧氧化生成相应的亚砜 38.

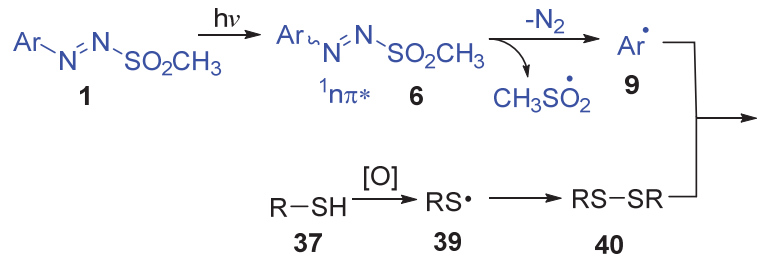

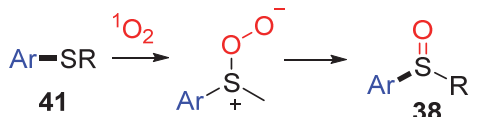

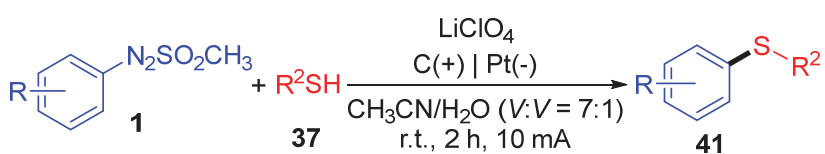

图式 20 合成不对称亚砜的反应机理

Scheme 20 Reaction mechanism for the synthesis of unsymmetrical sulfoxides

\section{2 电催化下芳基偶氮砜的芳基化反应}

近年来，电催化作为一种绿色的合成手段逐渐在有 机合成化学中得到应用 ${ }^{[29]} .2020$ 年，易文斌课题组 ${ }^{[30]}$ 首 次报道了电催化下利用芳基偶氮砜与硫酚或联硼酸频 那醇酯反应合成硫醚与芳基硼酸酯的方法(Schemes 21 和 22). 该方法利用 $\mathrm{C}(+) \mid \mathrm{Pt}(-)$ 作为反应电极，高氯酸 锂为电解质, 乙腈和水 $(V: V=7: 1)$ 作为反应的混合溶
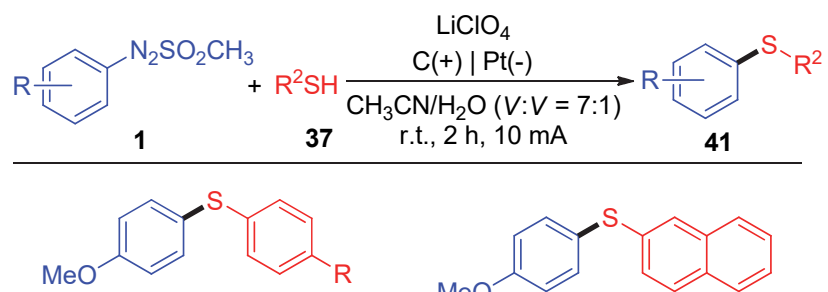

41a, $R=M e, 86 \% ; 41 b, R=E t, 81 \%$ 41c, $R=O M e, 91 \%$; 41d, $R=F, 87 \%$ 41e, $\mathrm{R}=\mathrm{Cl}, 85 \%$; 41f, $\mathrm{R}=\mathrm{CF}_{3}, 78 \%$

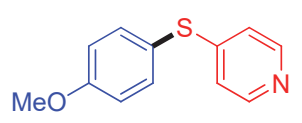

41h, $74 \%$<smiles>COc1ccc(Sc2nc3ccccc3s2)cc1</smiles>

$41 \mathrm{j}, 81 \%$

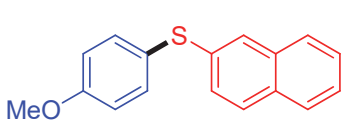

41g, $68 \%$<smiles>COc1ccc(Sc2c(F)c(F)c(F)c(F)c2F)cc1</smiles>

41i, $84 \%^{\mathrm{F}}$<smiles>COc1ccc(Sc2nnc(C)s2)cc1</smiles>

$41 \mathrm{k}, 80 \%$<smiles></smiles>

41I, $73 \%$<smiles>CCOC(=O)NC(CSc1ccc(OC)cc1)C(=O)OCC</smiles>

$41 \mathrm{~m}, 66 \%$

图式 21 电催化芳基偶氮砜与硫酚反应合成硫醚 Scheme 21 Electrocatalytic synthesis of thioethers from arylazo sulfones and thiols 

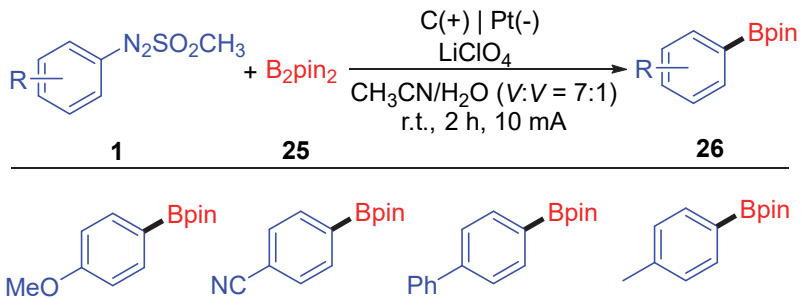

26a, $88 \%$

26e, $80 \%$
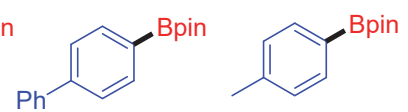

$\gamma^{\text {Bpin }}$

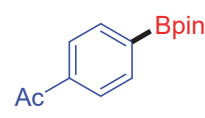

26i, $81 \%$ 26f $79 \%$

26g, $81 \%$

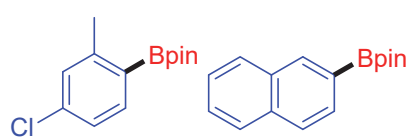

26j, $85 \%$ 26k, $78 \%$

图式 22 电催化芳基偶氮砜与联硼酸频那醇酯反应合成芳基 硼酸酯

Scheme 22 Electrocatalytic synthesis of aryl borates from arylazo sulfones and $\mathrm{B}_{2}$ pin 2

剂，在 $10 \mathrm{~mA}$ 电流下持续通电 $2 \mathrm{~h}$ 就可以合成相应的化 合物. 该反应不需要添加过渡金属催化剂、氧化剂或者 其他添加剂, 是一种非常新颖、绿色、高效的构建 $\mathrm{C}-\mathrm{S}$ 键和 $\mathrm{C}-\mathrm{B}$ 键的方法. 如 Scheme 21 所示, 各种非对称的 硫醚包括杂芳环及脂肪族硫醚都可以通过该合成方法 有效获得. 此外, 各种芳基偶氮砜在电催化下也都能以 令人满意的收率合成芳基联硼酸酯(Schemes 22).

\section{2 芳基偶氮砜的砜基化研究进展}

芳基偶氮砜在一定条件下可以裂解产生芳基和砜 基自由基，目前芳基化反应应用已经取得了重要进展. 相比之下, 芳基偶氮砜的砜基化反应研究起步较晚. 鉴 于有机砜化合物在有机合成和药物化学中的重要应用 价值, 有机砜的合成研究受到人们的广泛关注 ${ }^{[31]}$. 最 近，利用芳基偶氮砜作为的砜基前体构建有机砜化合物 也取得了一些进展.

$\beta$-羰基砜是一类极其重要的有机砜化合物, 其具有 广泛的生物活性如杀菌、消炎等. 同时, $\beta$-羰基砜也是一 种重要的有机合成中间体，可用来合成 $4 H$-吡喃、酮类、 石松碱、取代炔烃、丙二烯手性烯砜、喹啉衍生物以及 具有光化学活性的 $\beta$-差欮基砜等重要有机化合物 ${ }^{[32]} .2019$ 年, 魏伟课题组 ${ }^{[33]}$ 开发了一种利用可见光诱导下烯烃 与芳基偶氮砜和氧气的氧砜反应构建 $\beta$-羰基砜化合物 的方法. 该反应利用 $3 \mathrm{~W}$ Blue LED 灯作为反应光源, 以 空气为氧化剂, 无需使用光催化剂、金属试剂以及一些 酸、碱的添加剂, 在室温下即可完成各种 $\beta$-羰基砜化合 物的构建. 该反应具有良好的底物适用范围, 各种取代 的芳香族烯烃和杂芳环烯烃都可以适用于该反应. 此 外，多种烷基和芳基取代的偶氮砜也都能很好地兼容该 反应，以中等至较好的收率得到相应的 $\beta$-羰基砜化合物 (Scheme 23).

$$
\begin{aligned}
& \underset{\mathrm{ArN}_{2} \mathrm{SO}_{2} \mathrm{R}^{3}}{\stackrel{\mathrm{R}}{\mathbf{1}}}+\underset{\mathrm{R}^{2}}{\mathrm{R}}+\text { air }\left(\mathrm{O}_{2}\right) \underset{\text { 1,4-dioxane } / \mathrm{H}_{2} \mathrm{O}}{\stackrel{3 W}{\longrightarrow}} \\
& \mathrm{R}^{3}=\text { alkyl, aryl }
\end{aligned}
$$$$
\overbrace{\mathrm{R}^{2}}^{\mathrm{O}}
$$

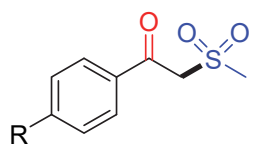

43a, $\mathrm{R}=\mathrm{H}, 80 \%$; 43b, $\mathrm{R}=\mathrm{Me}, 70 \%$;

43c, $\mathrm{R}={ }^{t} \mathrm{Bu}, 74 \%$; 43d, $\mathrm{R}=\mathrm{Cl}, 73 \%$

43e, $\mathrm{R}=\mathrm{Br}, 66 \%$; 43f, $\mathrm{R}=\mathrm{CF}_{3}, 46 \%$

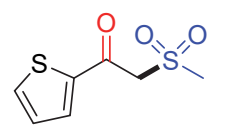

43h, $48 \%$

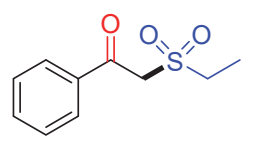

43i, $74 \%$

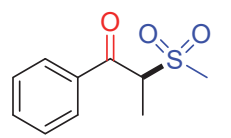

43g, $34 \%$

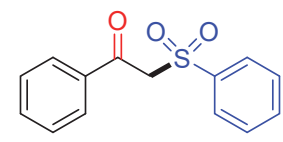

43j, $77 \%$<smiles>COc1ccc(S(=O)(=O)CC(=O)c2ccccc2)cc1</smiles>

43k, $66 \%$<smiles>O=C(CS(=O)(=O)c1ccc(F)cc1)c1ccccc1</smiles>

43I, $71 \%$
图式 23 光诱导烯烃与芳基偶氮砜和氧气反应构建 $\beta$-羰基砜 Scheme 23 Visible-light-induced reaction of alkenes with arylazo sulfones and dioxygen to construct $\beta$-keto sulfones

通过自由基捕捉实验和同位素标记实验，作者提出 了一种可能的自由基反应机理(Scheme 24). 首先, 在可 见光照射下芳基偶氮砜分解生成芳基自由基 9 和砜基自 由基 10, 同时释放出氮气. 随后, 芳基自由基与氧气和 水作用生成酚类化合物. 亲电性的砜自由基选择性加成 到富电子烯烃的 $\mathrm{C}=\mathrm{C}$ 上, 得到烷基自由基 44, 烷基自 由基 44 进一步被氧气捕捉，得到过氧自由基 45. 随后， 过氧自由基与磺酰基作用得到中间体 46. 最后，中间体 46 中 $\mathrm{C}-\mathrm{H}$ 键和 $\mathrm{O}-\mathrm{O}$ 键断裂, 脱去一分子磺酸得到 $\beta-$ 羰基砜化合物 43 .

随后，魏伟课题组 ${ }^{[34]}$ 又利用可见光诱导下芳基偶 氮砜与炔烃和氧气反应实现了一系列 $\beta$-羰基砜的构建. 该反应利用 1,4-二氧六环为反应溶剂, $3 \mathrm{~W}$ Blue LED 灯 为反应光源, 在 $50{ }^{\circ} \mathrm{C}$ 下一锅法合成了多类芳香族和脂 肪族的 $\beta$-羰基砜(Scheme 25). 该合成方法原料廉价易 得, 操作简单, 具有良好的底物和官能团相容性, 为 $\beta$ 羰基砜提供了一种有效构建方法.

可能的反应机理如 Scheme 26 所示，首先，在可见 光照射下, 芳基偶氮砜分解生成芳基自由基 9 和砜基自 由基 10. 紧接着, 砜基自由基 10 加成到富电子炔烃的 

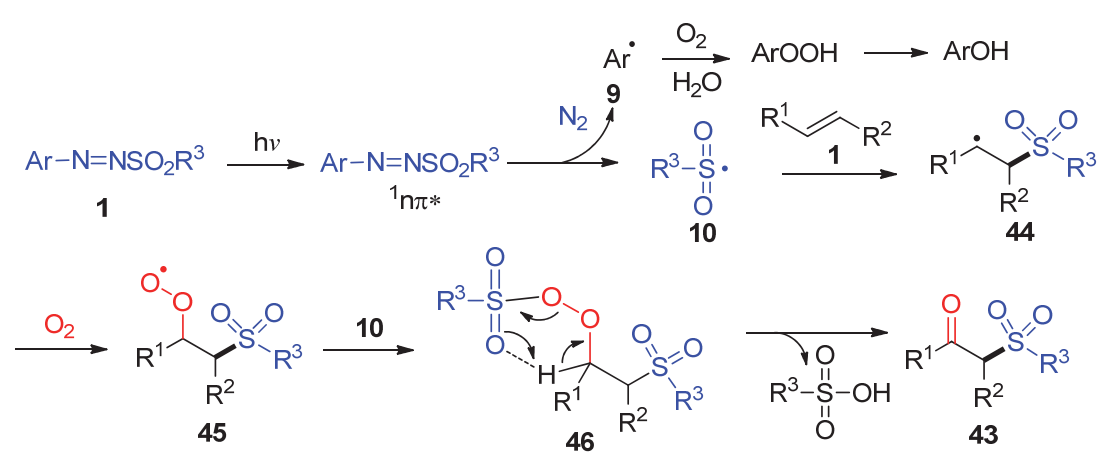

图式 24 烯烃氧砜化反应合成 $\beta$-羰基砜的反应机理

Scheme 24 Reaction mechanism for oxysulfonylation of alkenes to construct $\beta$-keto sulfones

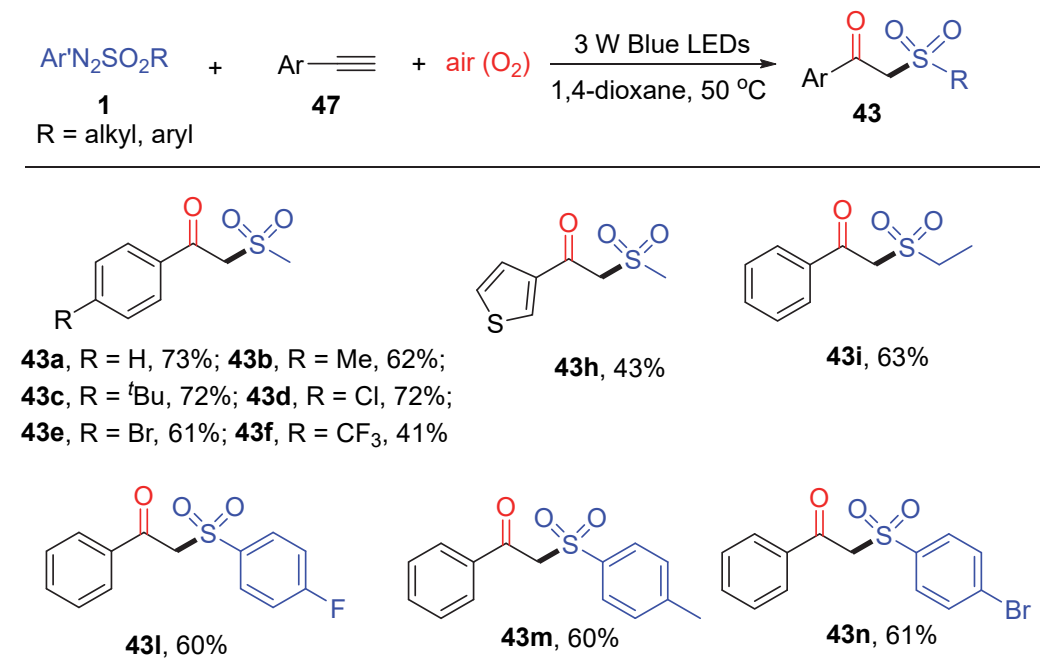

图式 25 光诱导芳基偶氮砜和炔烃反应构建 $\beta$-羰基砜

Scheme 25 Visible-light-induced reaction of arylazo sulfones and alkynes to construct $\beta$-keto sulfones

碳碳参键上, 得到烯基自由基 48. 烯基自由基 48 与氧 气结合, 得到烯基过氧自由基 49. 烯基过氧自由基与水 中的氢原子结合得到烯基过氧中间体 $\mathbf{5 0}$. 最后, 中间体 50 在砜自由基还原下生成了烯醇式中间体 51, 其经过 异构得到 $\beta$-羰基砜化合物 43 .

烯基砜化合物是一类重要的有机物结构单元, 广泛 存在于各类具有抗癌活性的天然产物、药物分子和医药
中间体中. 2020 年, Yadav 课题组 ${ }^{[35]}$ 发展了可见光诱导下 肉桂酸与芳基偶氮砜的脱羧砜化反应制备 $E$-烯基砜的 方法(Scheme 27). 他们利用芳基偶氮砜作为砜源, 在 3 $\mathrm{W}$ 蓝光照射下将砜基引入到肉桂酸分子上, 然后在碘 化钾和碳酸铯作用下脱羧合成 $E$-烯基砜. 该反应不需 要添加光催化剂和金属试剂, 在常温下即可完成肉桂酸 脱羧砜化反应.

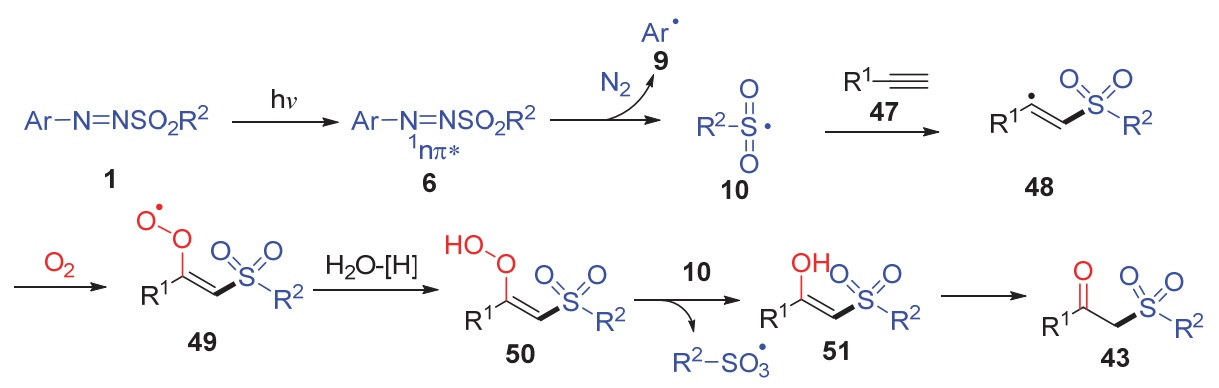

图式 26 炔烃氧砜化反应合成 $\beta$-羰基砜的反应机理

Scheme 26 Reaction mechanism for oxysulfonylation of alkynes to construct $\beta$-keto sulfones 


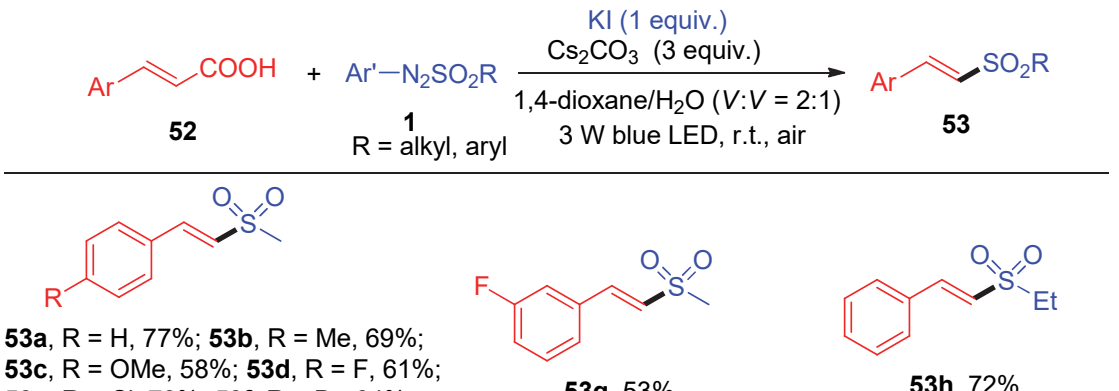

53c, $R=O M e, 58 \% ;$ 53d, $R=F, 61 \%$; 53e, $\mathrm{R}=\mathrm{Cl}, 70 \% ; \mathbf{5 3 f}, \mathrm{R}=\mathrm{Br}, 64 \%$

$53 \mathrm{~g}, 53 \%$<smiles>O=S(=O)(/C=C/c1ccccc1)c1ccc(Cl)cc1</smiles>

53j, $72 \%$<smiles>O=S(=O)(/C=C/c1ccccc1)c1ccc2ccccc2c1</smiles>

53k, $65 \%$

$53 \mathbf{i}, 79 \%$

图式 27 芳基偶氮砜和肉桂酸反应构建 $E$-烯基砜

Scheme 27 Reaction of arylazo sulfones and cinnamic acids to construct $(E)$-vinyl sulfones

其反应机理如 Scheme 28 所示, 在可见光照射下, 芳基偶氮砜分解生成了芳基自由基 9 和砜基自由基 $\mathbf{1 0}$. 芳基自由基 9 在氧气和水分子作用下生成了酚类化合 物. 肉桂酸分子在碳酸铯作用下脱掉氢离子生成羧酸 盐，随后砜基自由基 $\mathbf{1 0}$ 与碘离子在氧气作用下加成到 碳碳双键上，得到中间体 54. 最后，中间体 54 发生消除 反应脱去一分子 $\mathrm{CO}_{2}$ 和一分子 $\mathrm{I}^{-}$, 就得到了 $E$-烯基砜化 合物.

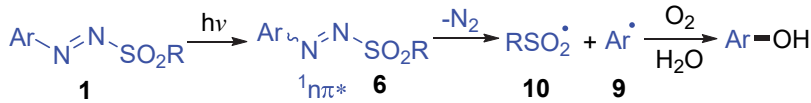

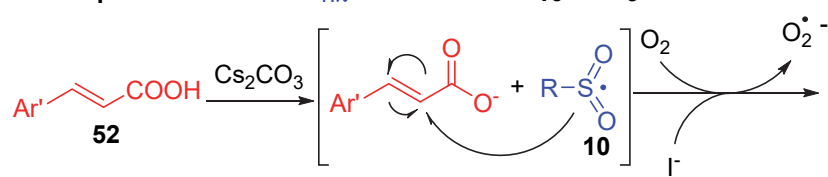

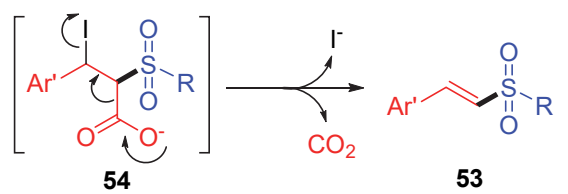

图式 28 芳基偶氮砜和肉桂酸砜化反应机理

Scheme 28 Reaction mechanism for sulfonylation of cinnamic acids with arylazo sulfones

$\gamma$-内酰胺是一类重要的含氮杂化合物, 广泛存在于 药物分子, 在有机合成化学中有着广泛应用. 2021 年, 魏伟课题组 ${ }^{[36]}$ 开发了一种非金属催化下利用芳基偶氮 砜与 1,6-烯炔构建砜代 $\gamma$-丁内酰胺化合物的方法 (Scheme 29). 该方法利用 1,4 -二氧六环/水 $(V: V=2: 1)$ 为反应溶剂, 无需任何催化剂, 在 $80{ }^{\circ} \mathrm{C}$ 温度下完成 1,6-烯炔分子的环化反应，以 $42 \% \sim 76 \%$ 的收率合成了 一系列砜代 $\gamma$-丁内酰胺.

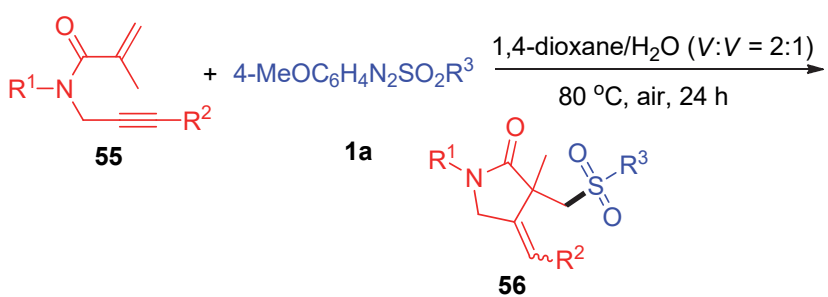

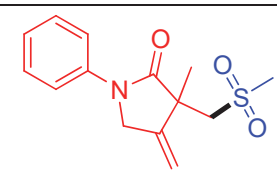

56a, $70 \%$

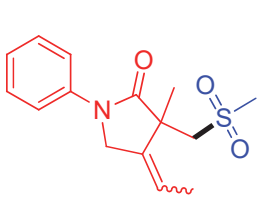

56g, $54 \%, Z: E=1.3: 1$

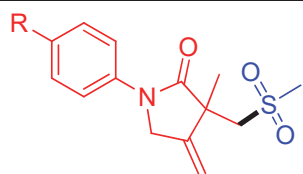

$\mathrm{R}=\mathrm{Me}, \mathbf{5 6 b}, 76 \% ; \mathrm{R}=\mathrm{OMe}, \mathbf{5 6 c}, 64 \%$ $R=F, 56 d, 74 \% ; R=C l, 56 e, 70 \%$ $\mathrm{R}=\mathrm{Br}, \mathbf{5 6 f}, 62 \%$

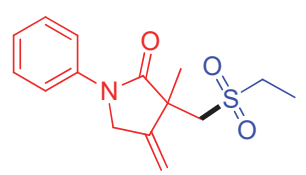

$56 h, 68 \%$<smiles>C=C1CN(c2ccccc2)C(=O)C1(C)CS(=O)(=O)c1ccc(C(F)(F)F)cc1</smiles>

$56 \mathbf{i}, 42 \%$

图式 29 芳基偶氮砜和 1,6-烯炔砜化/环化反应构建砜代 $\gamma$-丁 内酰胺

Scheme 29 Sulfonylation/cyclization of 1,6-enynes with arylazo sulfones leading to sulfonylated $\gamma$-butyrolactams

经历一系列控制实验后，作者提出来一个可能的反 应机理. 如 Scheme 30 所示：首先，芳基偶氮砜 1 在加热 条件下 $\mathrm{N}-\mathrm{S}$ 键均裂产生砜基自由基 10 和芳基自由基 9, 同时释放出氮气. 随后, 砜基自由基 10 选择性加成 到 $\mathrm{C}=\mathrm{C}$ 键上得到烷基自由基 56. 接着，烷基自由基 56 与碳碳三键进行分子内环化，得到烯基自由基 57. 最 
后, 中间体 57 从溶剂中夺取氢, 得到砜代 $\gamma$-丁内酰胺

56.
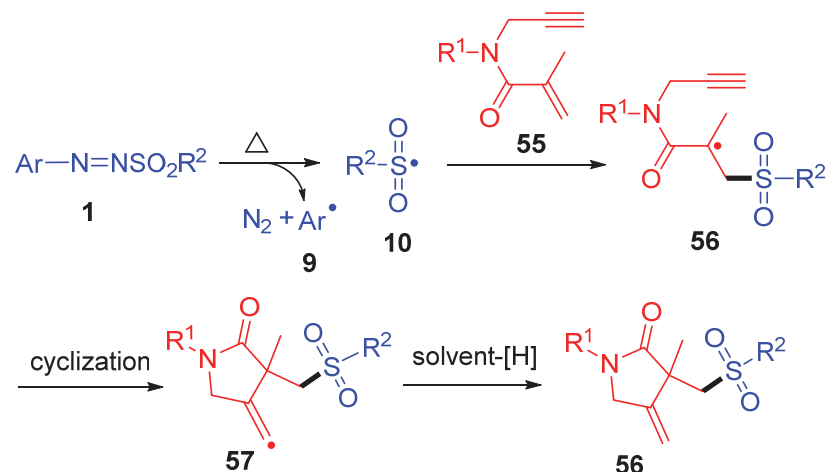

图式 30 合成砜代 $\gamma$-丁内酰胺的反应机理

Scheme 30 Reaction mechanism for the synthesis of sulfonylated $\gamma$-butyrolactams

\section{3 总结和展望}

综上所述, 芳基偶氮砜作为芳基化和砜基化试剂在 有机合成中越来越受到人们的关注. 与传统的合成方法 相比，芳基偶氮砜参与的芳基化和砜基化反应往往不需 要添加金属试剂、光催化剂、氧化剂或者其他添加剂, 在 比较温和的条件下就可以完成芳香环和有机砜化合物 的构建. 该类反应具有高效、绿色、安全等优点, 为一 些药物分子和功能材料提供了一种新的合成策略. 本文 主要从芳基偶氮砜裂解产生的芳基和砜基自由基诱导 方式出发, 分别从合成方法、底物范围和反应机理进行 总结和分析，阐述了芳基偶氮砜化的芳基化和砜基化应 用研究. 尽管芳基偶氮砜参与的芳基化和砜基化反应策 略已取得了一些进展, 但是目前该合成策略仍存在一些 局限性, 如反应底物过于简单, 合成方式的适用性较差. 相信随着研究的不断深入, 芳基偶氮砜对于复杂分子的 芳基和砜基化修饰方法一定会更加丰富.

\section{References}

[1] (a) Hanson, J. R. Nat. Prod. Rep. 1995, 12, 381.

(b) Tietze, L. F.; Raschke, T. Synlett 1995, 597.

[2] (a) Shiotani, S.; Kometani, T.; Mitsuhashi, K.; Nozawa, T.; Kurobe, A.; Futsukaichi, O. J. Med. Chem. 1976, 19, 803.

(b) Smethurst, P. W. R.; Forrest, W. H.; Hayden, J. Br. J. Anaesth. 1971, 43, 1129.

[3] (a) Roll, D. M.; Scheuer, P. J.; Matsumoto, G. K.; Clardy, J. J. Am. Chem. Soc. 1983, 105, 6177.

(b) Yano, H.; Nakanishi, S. J. Biol. Chem. 1993, 268, 25846.

[4] Yuan, Z. Z.; Dai, Q.; Qiao, L.; Zhao, Y. Y.; Zhang, H. M.; Li, X. F. J. Membr. Sci. 2017, 541,465 .

[5] (a) Mack, D. J.; Njardarson, J. T. Angew. Chem., Int. Ed. 2013, 52, 1543 .

(b) Wang, N. Z.; Saidhareddy, P.; Jiang, X. F. Nat. Prod. Rep. 2020, $37,246$.

[6] Yazdanyar, S.; Boer, J.; Ingvarsson, G.; Szepietowski, J. C.; Jemec, G. B. E. Dermatology 2011, 222, 342.

[7] Li, P.; Hu, D. Y.; Xie, D. D.; Chen, J. X.; Jin, L. H.; Song, B. A. J.
Agric. Food Chem. 2018, 66, 3093.

[8] (a) Williams, T. M.; Ciccarone, T. M.; MacTough, S. C.; Rooney, C. S.; Balani, S. K.; Condra, J. H.; Emini, E. A.; Goldman, M. E.; Greenlee, W. J. J. Med. Chem. 1993, 36, 1291.

(b) Artico, M.; Silvestri, R.; Massa, S.; Loi, A. G.; Corrias, S.; Piras, G.; Colla, P. L. J. Med. Chem. 1996, 39, 522.

[9] Kamigta, N.; Kobayashi, M. Sulfur Rep. 1982, 2, 87.

[10] (a) Kice, J. L.; Gabrielsen, R. S. J. Org. Chem. 1970, 35, 1004.

(b) Kobayashi, M.; Gotoh, M.; Minato, H. J. Org. Chem. 1975, 40 , 140 .

(c) Evers, M. J.; Christiaens, L. E.; Guillaume, M. R.; Renson, M. J. J. Org. Chem. 1985, 50, 1779.

(d) Sapountzis, I.; Knochel, P. Angew. Chem., Int. Ed. 2004, 43, 897.

(e) Qiu, D.; Lian, C.; Mao, J.; Fagnoni, M.; Protti, S. J. Org. Chem. 2020, 85,12813

[11] (a) Gaikwad, D. S.; Pore, D. M. Synlett 2012, 23, 2631.

(b) Wang, L.; Bao, P.; Liu, W.; Liu, S.; Hu, C.; Yue, H.; Yang, D.; Wei, W. Chin. J. Org. Chem. 2018, 38, 3189 (in Chinese). (王雷雷, 鲍鹏丽, 刘维伟, 刘思形, 胡昌松, 岳会兰, 杨道山, 魏伟, 有机化学, 2018, 38, 3189.)

[12] (a) Shen, Q. L.; Ogata, T.; Hartwig, J. F. J. Am. Chem. Soc. 2008, 130, 6586 .

(b) Arockiam, P. B.; Fischmeister, C.; Bruneau, C.; Dixneuf, P. H. Green Chem. 2013, 15, 67.

(c) Prades, A.; Poyatos, M.; Peris, E. Adv. Synth. Catal. 2010, 352, 1155.

[13] (a) Rao Volla, C. M.; Vogel, P. Angew. Chem., Int. Ed. 2008, 47, 1305.

(b) Zeng, X. M.; Ilies, L.; Nakamura, E. J. Am. Chem. Soc. 2011, 133, 17638 .

[14] (a) Duong, H. A.; Gilligan, R. E.; Cooke, M. L.; Phipps, R. J.; Gaunt, M. J. Angew. Chem., Int. Ed. 2011, 50, 463.

(b) Vásquez-Céspedes, S.; Holtkamp, M.; Karst, U.; Glorius, F. Synlett 2017, 28, 2759.

[15] (a) Kumar, M. R.; Park, K.; Lee. S. Adv. Synth. Catal. 2010, 352, 3255 .

(b) Hodgetts, K. J.; Kershaw, M. T. Org. Lett. 2003, 5, 2911.

[16] (a) Chen, J.-R.; Hu, X.-Q.; Lu, L.-Q.; Xiao, W.-J. Chem. Soc. Rev. 2016, 45, 2044.

(b) Yu, X.-Y.; Chen, J.-R.; Xiao, W.-J. Chem. Rev. 2021, 121, 506.

(c) He, W.-B.; Gao, L.-Q.; Chen, X.-J.; Wu, Z.-L.; Huang, Y.; Cao, Z.; Xu, X.-H.; He, W.-M. Chin. Chem. Lett. 2020, 31, 1895.

(d) Liu, Q.; Wang, L.; Yue, H.; Li, J.-S.; Luo, Z.; Wei, W. Green Chem. 2019, 21, 1609.

(e) Gan, Z.; Li, G.; Yang, X.; Yan, Q.; Xu, G.; Li, G.; Jiang, Y.-Y.; Yang, D. Sci. China: Chem. 2020, 63, 1652.

(f) Chen, J.-R.; Yan, D.-M.; Wei, Q.; Xiao, W.-J. ChemPhotoChem 2017, 1,148

(g) Liu, R.; Liu, Q.; Meng, H.; Ding, H.; Hao, J.; Ji, Z.; Yue, H.; Wei, W. Org. Chem. Front. 2021, 8, 1970.

(h) Ye, H.; Xiao, C.; Lu, L. Chin. J. Org. Chem. 2018, 38, 1897 (in Chinese).

(叶辉, 肖㙂, 陆良秋, 有机化学, 2018, 38, 1897.)

[17] Crespi, S.; Protti, S.; Fagnoni, M. J. Org. Chem. 2016, 81, 9612.

[18] Dossena, A.; Sampaolesi, S.; Palmieri, A.; Protti, S.; Fagnoni, M. J. Org. Chem. 2017, 82, 10687.

[19] Sauer, C.; Liu, Y.; Nisi, A. D.; Protti, S.; Fagnoni, M.; Bandini, M. ChemCatChem 2017, 9, 4456.

[20] Malacarne, M.; Protti, S.; Fagnoni, M. Adv. Synth. Catal. 2017, $359,3826$.

[21] Onuigbo, L.; Raviola, C.; Fonzo, A. D.; Protti, S.; Fagnoni, M. Eur. J. Org. Chem. 2018, 2018, 5297.

[22] Xu, Y. L.; Yang, X. Y.; Fang, H. J. Org. Chem. 2018, 83, 12831.

[23] (a) Lawrence, D. S.; Copper, J. E.; Smith, C. D. J. Med. Chem. 2001, 44, 594.

(b) Willardsen, J. A.; Dudley, D. A.; Cody, W. L.; Chi, L.; McClanahan, T. B.; Mertz, T. E.; Potoczak, R. E.; Narasimhan, L. 
S.; Holland, D. R.; Rapundalo, S. T.; Edmunds, J. J. J. Med. Chem. 2004, 47, 4089.

(c) Galal, S. A.; Khairat, S. H. M.; Ragab, F. A. F.; Abdelsamie, A. S.; Ali, M. M.; Soliman, S. M.; Mortier, J.; Wolber, G.; El Diwani, H. I. Eur. J. Med. Chem. 2014, 86, 122.

[24] (a) Yuan, J.; Zhu, J.; Fu, J.; Yang, L.; Xiao, Y.; Mao, P.; Dua, X.; Qu, L. Org. Chem. Front. 2019, 6, 925.

(b) Wei, W.; Wang, L.; Bao, P.; Shao, Y.; Yue, H.; Yang, D.; Yang, X.; Zhao, X.; Wang, H. Org. Lett. 2018, 20, 7125.

(d) Meng, N.; Lü, Y.; Liu, Q.; Liu, R.; Zhao, X.; Wei, W. Chin. Chem. Lett. 2021, 32, 258.

(e) Bao, P.; Liu, F.; Lü, Y.; Yue, H.; Li, J.-S.; Wei, W. Org. Chem. Front. 2020, 7, 492 .

(h) Shi, J.; Wei, W. Chin. J. Org. Chem. 2020, 40, 2170 (in Chinese). (时建伟, 魏伟, 有机化学, 2020, 40, 2170.)

[25] Jung, H. I.; Lee, J. H.; Kim, D. Y. Bull. Korean Chem. Soc. 2018, $39,1003$.

[26] Lian, C.; Yue, G. L.; Mao, J. S.; Liu, D. Y.; Ding, Y.; Liu, Z. R.; Qiu, D.; Zhao, X.; Lu, K.; Fagnoni, M.; Protti, S. Org. Lett. 2019, $21,5187$.

[27] Qiu, D.; Lian, C.; Mao, J. S.; Ding, Y.; Liu, Z. R.; Wei, L. Y.; Fagnoni, M.; Protti, S. Adv. Synth. Catal. 2019, 361, 5239.

[28] Liu, Q. S.; Wang, L. L.; Yue, H. L.; Li, J. S.; Luo, Z. D.; Wei, W. Green Chem. 2019, 21, 1609.

[29] (a) Zhang, L.; Niu, C.; Yang, X.; Qin, H.; Yang, J.; Wen, J.; Wang, H. Chin. J. Org. Chem. 2020, 40, 1117 (in Chinese). (张龙菲, 牛聪, 杨晓婷, 秦宏云, 杨建静, 文江伟, 王桦, 有机 化学, 2020, 40, 1117.)

(b) Feng, E.; Hou, Z.; Xu, H. Chin. J. Org. Chem. 2019, 39, 1424 (in Chinese).

(冯恩祺, 侯中伟, 徐海超, 有机化学, 2019, 39, 1424.)

(c) Li, M.; Wang, R.; Hao, W.; Jiang, B. Chin. J. Org. Chem. 2020, 40, 1540 (in Chinese).
(李梦帆，王榕，郝文娟，姜波，有机化学，2019，39,1540.)

(d) Meng, W.; Xu, K.; Guo, B.; Zeng, C. Chin. J. Org. Chem. 2021, 41, 2621 (in Chinese).

(孟薇, 徐坤, 郭兵兵, 曾程初, 有机化学, 2021, 41, 2621.)

(e) Wu, Y.; Chen, J.-Y.; Ning, J.; Jiang, X.; Deng, J.; Deng, Y.; Xu, R.; He, W.-M. Green Chem. 2021, 23, 3950.

(f) Chen, J.-Y.; Zhong, C.-T.; Gui, Q.-W.; Zhou, Y.-M.; Fang, Y.-Y.; Liu, K.-J.; Lin, Y.-W.; Cao, Z.; He, W.-M. Chin. Chem. Lett. 2021 $32,475$.

[30] Wang, R. K.; Chen, F. M.; Jiang, L. Q.; Yi, W. B. Adv. Synth. Catal. 2020, 363, 1904.

[31] (a) Li, Y.; Wan, J.-P. Chin. J. Org. Chem. 2020, 40, 3889 (in Chinese).

(李毅, 万结平, 有机化学, $\mathbf{2 0 2 0}, 40,3889$.)

(b) Liu, Q.; Lü, Y.; Liu, R.; Zhao, X.; Wang, J.; Wei, W. Chin. Chem. Lett. 2021, 32, 136.

(c) Bao, W.-H.; Wang, Z.; Tang, X.; Zhang, Y.-F.; Tan, J.-X.; Zhu, Q.; Cao, Z.; Lin, Y.-W.; He, W.-M. Chin. Chem. Lett. 2019, 30, 2259 .

(d) Lia, G.-H.; Han, Q.-Q.; Sun, Y.-Y.; Chen, D.-M.; Wang, Z.-L.; Xu, X.-M.; Yu, X.-Y. Chin. Chem. Lett. 2020, 31, 3255.

(e) Wang, L.; Zhang, M.; Zhang, Y.; Liu, Q.; Zhao, X.; Li, J.-S.; Luo, Z.; Wei, W. Chin. Chem. Lett. 2020, 31, 67.

[32] Wei, W.; Liu, C.; Yang, D.; Wen, J.; You, J.; Suo, Y.; Wang, H. Chem. Commun. 2013, 49, 10239.

[33] Liu, Q. S.; Liu, F.; Yue, H. L.; Zhao, X. H.; Li, J. S.; Wei, W. Adv. Synth. Catal. 2019, 361, 5277.

[34] Lü, Y. F.; Liu, Q. S.; Liu, F.; Yue, H. L.; Li, J. S.; Wei, W. Tetrahedron Lett. 2020, 61, 151335.

[35] Chawla, R.; Jaiswal. S.; Dutta, P. K.; Yadav, L. D. S. Tetrahedron Lett. 2020, 61, 151898.

[36] Liu, Q. S.; Lü, Y. F.; Liu, R. S.; Zhao, X. H.; Wang, J. W.; Wei, W. Chin. Chem. Lett. 2021, 32, 136. 